\title{
Stenting as porous media in anatomically accurate geometries. A comparison of models and spatial heterogeneity
}

\author{
Nicolás Dazeo $^{\mathrm{a}, *}$, Javier Dottori ${ }^{\mathrm{a}}$, Gustavo Boroni ${ }^{\mathrm{a}}$, Ana Paula Narata ${ }^{\mathrm{b}}$, Ignacio Larrabide ${ }^{\mathrm{a}}$ \\ a Pladema - CONICET, Universidad Nacional del Centro de la Provincia de Buenos Aires, Buenos Aires, Argentina \\ ${ }^{\mathrm{b}}$ University Hospital of Tours, UMR Imagerie et Cerveau, Inserm U930, Université François-Rabelais, Tours, France
}

\section{ART ICLE IN F O}

\section{Article history:}

Accepted 8 July 2020

Available online xxx

\section{Keywords}

CFD

Flow diverter

Modelling

Porous medium

\begin{abstract}
A B S T R A C T
Modelling intracranial aneurysm blood flow after flow diverter treatment has proven to be of great scientific and clinical interest. One of the reasons for not having CFD as an everyday clinical tool yet is the time required to set-up such simulations plus the required computational time. The speed-up of these simulations can have a considerable impact during treatment planning and device selection. Modelling flow diverters as a porous medium (PM) can considerably improve the computational time. Many models have been presented in literature, but quantitative comparisons between models are scarce.In this study, the untreated case, the explicit definition of the flow diverter wires as no-slip boundary condition and five different porous medium models were chosen for comparison, and evaluated on intracranial aneurysm of 14 patients with different shapes, sizes, and locations. CFD simulations were made using finite volume method on steady flow conditions. Velocities, kinetic energy, wall shear stress, and computational time were assessed for each model. Then, all models are compared against the no-slip boundary condition using non parametric Kolmogorov-Smirnov test.The model with least performance showed a mean K-S statistic of 0.31 and deviance of 0.2 , while the model with best values always gave K-S statistics below 0.2. Kinetic energy between PM models varied between an over estimation of $218.3 \%$ and an under estimation of $73.06 \%$. Also, speedups were between $4.75 \mathrm{x}$ and 5.3x (stdev: 0.38x and 0.15x) when using PM models.Flow diverters can be simulated with PM with a good agreement to standard CFD simulations were FD wires are represented with no-slip boundary condition in less than a quarter of the time. Best results were obtained on PM models based on geometrical properties, in particular, when using a heterogeneous medium based on equations for flat rhomboidal wire frames.
\end{abstract}

\section{Introduction}

Intracranial aneurysms are malformations in cerebral vessel walls. To treat them, intravascular devices, such as Flow Diverter (FD) stents, are deployed in the parent vessel affected by the aneurysm thus modifying local hemodynamics and redirecting the flow away from the aneurysm. Computational Fluid Dynamics (CFD) is a non invasive way to simulate treatment effect on local hemodynamics. To model a FD wire screen in a CFD finite volume mesh, volumetric element faces are locally refined and snapped to the nearest wire's surface, and a no-slip boundary conditions is set over the surface of the wire. This FD modelling method, subsequently recalled as Boundary Condition Method (BCM), requires a 2 orders of magnitude finer mesh around each FD wire than the rest of the vessel. This is because of the difference of size

\footnotetext{
* Corresponding author.

E-mail address: ndazeo@exa.unicen.edu.ar (N. Dazeo)
}

between the FD wires and the parent vessel lumen, and the need for a finer mesh resolution to capture local flow behaviour. This mesh refinement leads to a considerable increase in the computational load. To mitigate the increased computational cost associated to a finer mesh, Augsburger et al. proposed a coefficient estimation method for modelling FD as Porous Medium (PM) (Augsburger et al., 2011). Their model is based on a quadratic fit of pressure drop across the FD screen vs velocity obtained from simulations of flow across an equivalent FD wire screen on an ideal channel. Later on, other authors proposed new models based on FD wire screen geometrical properties to avoid additional simulations (Morales and Bonnefous, 2014; Raschi et al., 2014; Dazeo et al., 2017; Yadollahi-Farsani et al., 2019; Li et al., 2019). In 2018, Dazeo et al. compared different PM models in idealised channels, measuring pressure drop and average velocity in the PM zone (Dazeo et al., 2018a). Those variables are representative in ideal geometries, but not in anatomically accurate ones. In this work, a comparison between FD models in patient-specific geometries is implemented and assessed. 


\section{Materials and methods}

\subsection{Image acquisition and 3D modelling}

Fourteen patients with intracranial aneurysm of different shapes, sizes, and locations were chosen for the study. The data acquisition protocol of this retrospective study was approved by the University Hospitals of Tours, Toulouse, France, institutional review board. Pre-treatment anatomical models were generated from 3D Rotational Angiography (3DRA) images using an INNOVA $3131 \mathrm{IQ}^{\mathrm{TM}}$ (General Electric Healthcare, Milwaukee, USA, $n=47$ ). Vascular anatomy was extracted from 3DRA images with voxel size of $0.208 \mathrm{~mm} \times 0.208 \mathrm{~mm} \times 0.208 \mathrm{~mm}$ using threshold-based segmentation (Moyano et al., 2017) Threshold values were chosen by an expert in neurovascular angiography to best fit the patient's anatomy depending on contrast density and image quality. When selecting the threshold value, both the treated vessel and aneurysm, were considered as the priority to achieve a high quality reconstruction of the local vascular anatomy (Larrabide et al., 2012b). Some cases needed additional post-processing to achieve a good quality representation of the anatomy, leading to a post-processing rate similar to previous studies (Narata et al., 2018). Before preforming the CFD simulation, each 3D model was then visually validated by expert interventional neuroradiologists (INR). Streamline visualisation of CFD simulations was used to classify them into shear-drive and inertia-driven.

\subsection{Stent deployment}

For each patient's geometry, the FD stent was deployed virtually in accordance with clinical procedures and using the process described by Larrabide et al. (2012a). The FD model represents a typical Surpass FD (Stryker, Kalamazoo, MI, USA) with 48 wires of $5 \times 10^{-5} \mathrm{~m}$ width. The FD diameter was set to fit the parent vessel lumen diameter for a correct apposition of the FD to the arterial wall. For the same patient-specific geometry several analyses were run, with and without stent, and for different flow conditions.

\subsection{Simulations}

The open source Finite Volume software OpenFOAM was used for CFD simulations. A steady state solver capable of modelling heterogeneous PM was implemented and used in all the experiments (Jasak et al., 2007; Dazeo et al., 2018b).

Morales et al. showed that local differences between Newtonian and non Newtonian fluids in coiled intracranial aneurysm are not sufficient to alter the main flow characteristics (Morales et al., 2013). With this in mind, and because velocities in aneurysms treated with coils are smaller than velocities in aneurysms treated with FDs, blood behaviour can be safely assumed as Newtonian in the former. Viscosity was considered as $\mu=3.86835 \times 10^{-03} \mathrm{~Pa} \cdot \mathrm{s}$ and density $\rho=1025 \mathrm{~kg} / \mathrm{m}^{3}$ to approximate physiological range of human blood. Each patient's inlet flow rate $(q)$ was set using the following equation proposed by Reneman et al. (2006), namely:

$q=\frac{\tau \pi r^{3} \rho}{4 \mu}$,

were $r$ is the inlet radius and $\tau$ is the wall shear stress with a value of 1.5 Pa, following the methodology proposed by Castro et al. (2009). Inlet of the geometries was extended to gradually match a circular cross section. Inlet boundary condition was set, for all models, at a similar location of the internal carotid artery. Pressure was set to $0 \mathrm{~Pa}$ in all the outlets.

\subsection{Porous Medium models}

The goal of this work is to assess how different PM models described in the literature perform when modelling FD in real geometries. To model a PM region within the CFD simulation, a Brinkman-Forchheimer term is added to the momentum equation (Dazeo et al., 2018b):

$\rho(\mathbf{U} \cdot \nabla) \mathbf{U}=-\nabla P+\mu \nabla^{2} \mathbf{U}-\left(\mu \mathbf{D}+\frac{1}{2} \rho \mathbf{U} \cdot \mathbf{F}\right) \cdot \mathbf{U}$,

where $\mathbf{U}$ is the velocity, $P$ is the pressure, $\mathbf{D}$ is the Darcy tensor and $\mathbf{F}$ is the Forchheimer tensor. Darcy and Forchheimer tensors have non-zero values only in the region of the FD screen (Fig. 1a). As described below, each model proposes a different way to derive the values of $\mathbf{D}$ and $\mathbf{F}$.

\subsubsection{Augsburger (A)}

Augsburguer et al. modelled the FD screen by the addition of a viscous and an inertial loss to the momentum equation (Augsburger et al., 2011). These terms contribute to the computation of the pressure gradient across a porous cell. The FD screen is modelled as a homogeneous anisotropic PM region. This implementation requires two coefficients, both for the viscous and the inertial terms, for the two principal orthogonal directions. These coefficients need to be computed independently for each patient as they depend on the portion of the FD across the neck (i.e., its final shape) once placed inside the patient. In their work, the authors obtain those coefficients via BCM simulations, by placing the portion of the FD that covers the aneurysm neck in an ideally shaped channel with different velocities.

Simulations are done on a pipe of $1.128 \times 10^{-3} \mathrm{~m}$ in diameter to assess perpendicular flow, and on a $0.9 \times 10^{-3} \mathrm{~m}$ per $0.2 \times 10^{-3} \mathrm{~m}$ rectangular pipe to assess parallel flow. The wire screen is then introduced in both pipes, as shown in Fig. 1c with a no-slip boundary condition. The authors do not provide details about lateral boundaries nor channel length and, therefore, slip conditions for the lateral patches and a channel length of $5 \times 10^{-3} \mathrm{~m}$ were set. No-slip patches would lead to an unwanted extra pressure drop.

Then, the relation between velocity and pressure drop for both configurations is computed as:

$\Delta P=d \mathbf{U}+f \mathbf{U} \cdot \mathbf{U}$,

where $\mathbf{U}$ is the velocity vector, $d$ is the viscous (Darcy) term, and $f$ is the inertial (Forchheimer) term. Finally, tensors $\mathbf{D}$ and $\mathbf{F}$ are created from perpendicular and tangential coefficients. These tensors are aligned to the perpendicular an tangential directions of the FD screen.

\subsubsection{Raschi (R1)}

Raschi et al. used hydraulic resistance equations proposed by Idelchik for wire screens of multiple shapes and sizes to model FD stents (Raschi et al., 2014; Idelchik and Fried, 1986). In his work, Idelchik described the general use of PM model to represent flow passing by regions with different shaped and geometries. These equations calculate Darcy and Forchheimer coefficients from the occupied volume and wetted surface of the wire screen based on its geometrical properties:

$d=\frac{11 \mu}{d_{h} \Delta L}$,

$f=\frac{\rho}{2 \Delta L}\left(1.3(1-\beta)+\left(\beta^{-1}-1\right)^{2}\right)$,

where $\mu$ and $\rho$ are the dynamic viscosity and the density of the fluid, and $\Delta L$ is the width of the porous region. The hydraulic diameter $d_{h}$, is 




(a)
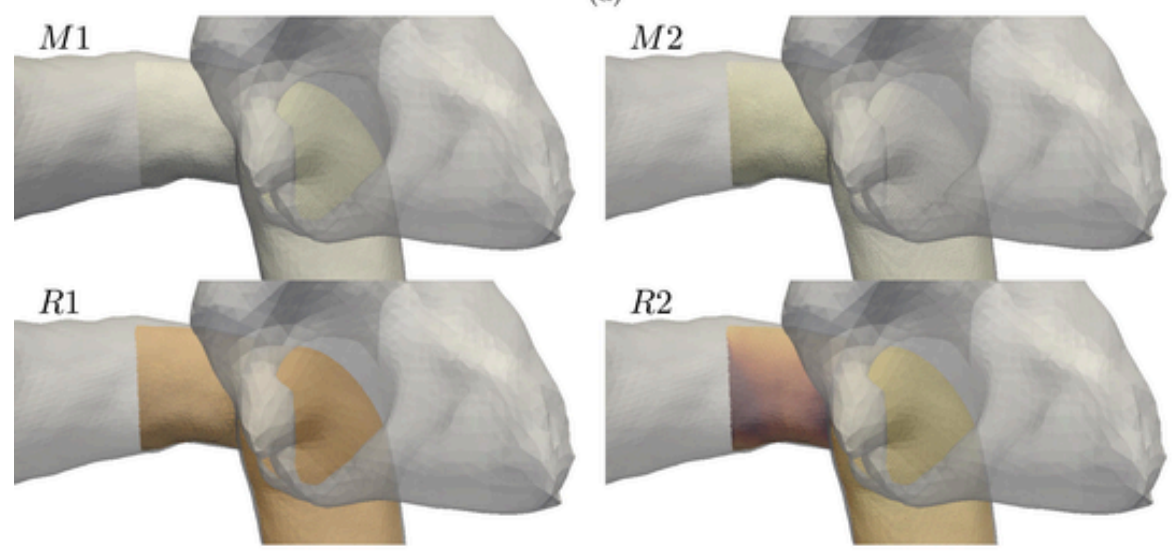

(b)

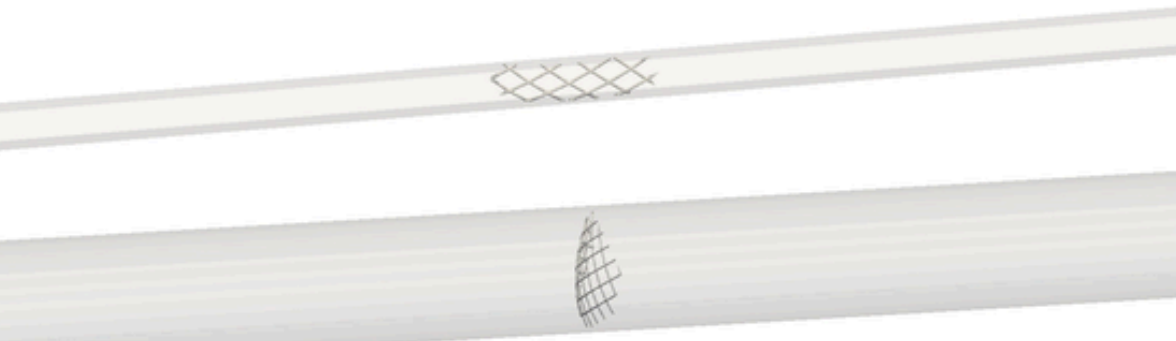

(c)

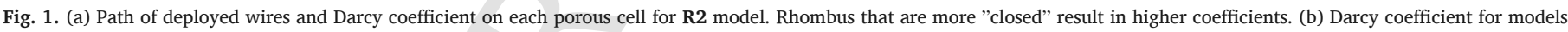


(below) placement of the stent in the test volumes.

defined as the ratio of four times the void volume $V_{\text {void }}$ to the wetted surface area $A_{w}$ :

$$
\begin{gathered}
d_{h}=\frac{4 V_{\text {void }}}{A_{w}} \\
=\frac{4 l_{x} l_{y} r-2 \pi r^{2}\left(l+\frac{1}{2} l_{0}+\frac{1}{2} l_{1}\right)}{\pi r\left(l+\frac{1}{2} l_{0}+\frac{1}{2} l_{1}\right)} \\
=\frac{4 l_{x} l_{y}}{\pi\left(l+\frac{1}{2} l_{0}+\frac{1}{2} l_{1}\right)}-2 r,
\end{gathered}
$$

being $r$ the strut radio obtained as $r=t / 2$ and $\beta$ the cross-sectional porosity, defined as the open area $A_{\text {open }}$ by the total area $A_{\text {total }}$ :

$$
\beta=\frac{A_{\text {open }}}{A_{\text {total }}}=\frac{l_{x}^{\prime} l_{y}^{\prime}}{l_{x} l_{y}} .
$$

Finally, $l, l_{x}, l_{y}, l_{0}, l_{1}, l_{x}^{\prime}, l_{y}^{\prime}$ are geometrical variables that can be obtained from the deployed FD (Fig. 2).

\subsubsection{Morales (M1)}

Morales et. al. proposed an analytical model for PM coefficients based on statistical properties of a deployed FD (Morales and Bonnefous, 2014). Authors consider invariant Darcy and Forchheimer coefficients obtained from an average FD screen porosity $\beta$, namely:

$d=\frac{2 \mu \pi^{2}\left(1-\beta^{-1}\right)^{2}}{\beta^{-3} t^{2}}$,

$f=\frac{\sqrt{2} \rho 1.2 \pi\left(1-\beta^{-1}\right)}{\beta^{-2} t}$,

where $t$ is the wire width. 


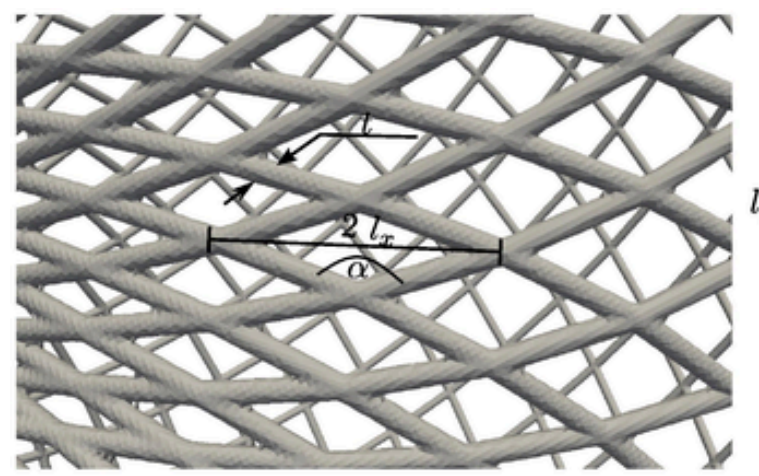

(a)

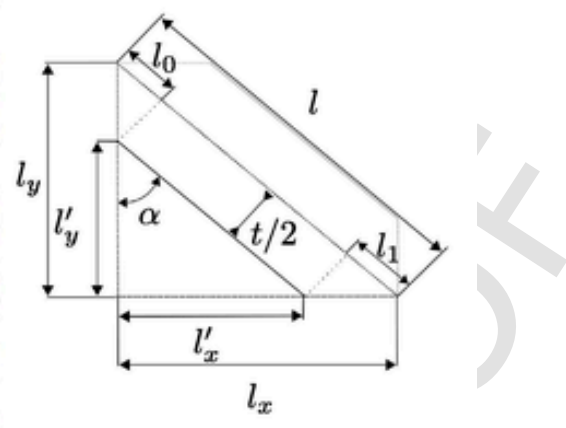

(b)

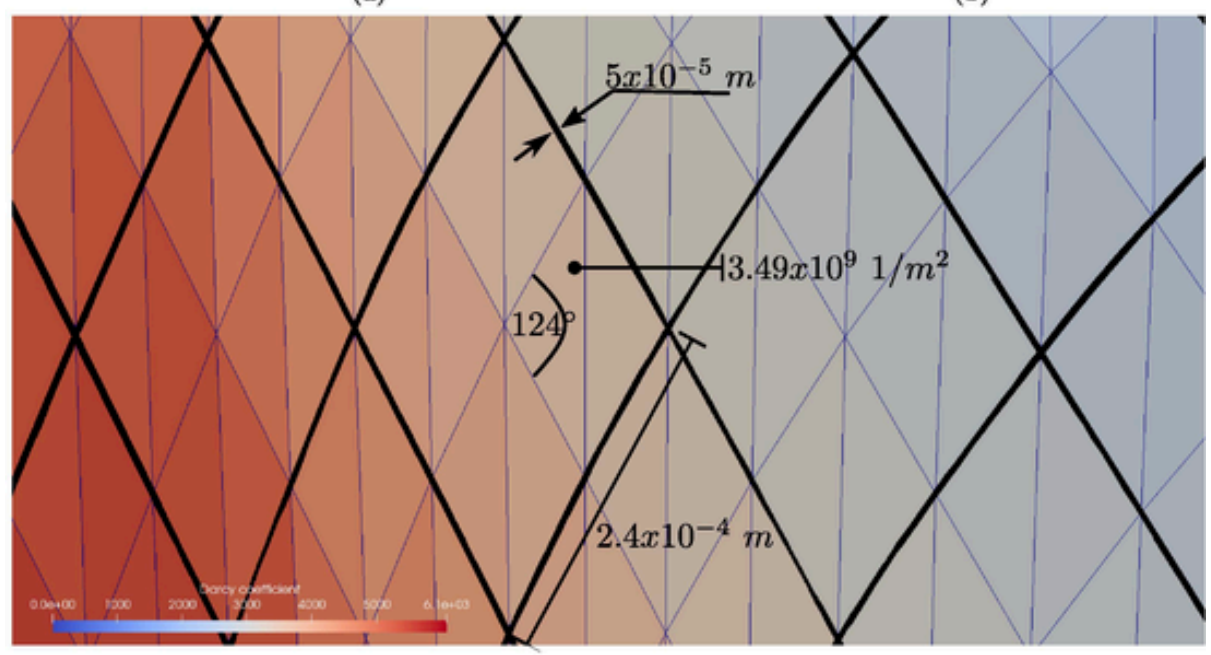

(c)

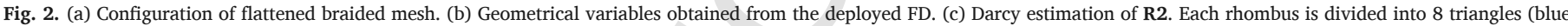

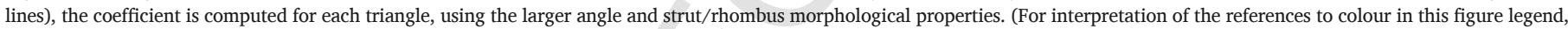
the reader is referred to the web version of this article.)

\subsection{Heterogeneous variants (R2 and $\mathbf{M} 2)$}

More recently, Farsani et al. proposed using different coefficients for each PM finite volume element considering the local FD screen configuration, thus creating an heterogeneous variant (Yadollahi-Farsani et al., 2019). Following this approach, both models based on FD screen geometrical properties (R1 and M1) were adapted to incorporate the local heterogeneity considering variations in the local screen geometry when calculating the corresponding coefficients. This can be seen in Fig. 1b. To achieve a smoother discretization of the PM, each rhombus is divided into eight triangles as shown in Fig. 2c (blue lines). Darcy and Forchheimer coefficients are computed for each triangle, using the larger angle and the local wire/rhombus morphology. For example, as shown in Fig. 2c, for a triangle with angle $124^{\circ}$, a wire width of $t=5 \times 10^{-5} \mathrm{~m}$ and a length of $2.4 \times 10^{-4} \mathrm{~m}$, the corresponding hydraulic diameter and Darcy coefficient for $\mathbf{R} \mathbf{2}$ are given by:

$$
\begin{gathered}
d_{h}=6.29 \times 10^{-5}, \\
\frac{d}{\mu}=\frac{6.29 \times 10^{-5}}{5 \times 10^{-5}}=3.49 \times 10^{9} .
\end{gathered}
$$

This results in two heterogeneous PM variants (R2 and M2), which were also studied.

\subsection{CFD variables studied}

Steady state simulations provide converged pressure and velocity fields. These variables need to be compared between models to properly assess differences between PM models. Because velocity and pressure are coupled, i.e. the first imply changes in the former, only velocity fields were directly compared and presented in the manuscript.

Velocity is a volumetric, 3 component, vector field that describes a fluid in motion. The velocity at the aneurysm sac is typically dumped after placement of the FD stent is deployed (Larrabide et al., 2013). This effect is expected to be stronger when the wire screen is simulated using PM. To compare the velocity fields between PM and BCM, velocity magnitude is probed over a uniformly distributed cloud of points in the aneurysm sac.

When analysing hemodynamic properties of an aneurysm, there is a special interest in the aneurysm dome and wall, as this is the region where rupture and thrombus formation typically occur. Therefore, a cloud of uniformly distributed points was created within each aneurysm and then is partitioned in regions in two different ways. The first partition considers the distance to the aneurysm wall, considering in the wall region the $25 \%$ of the points closer to it, and the core region with the remaining points (Fig. 3a). The second partition separates the aneurysm in three by the distance to the neck geometrical center: the neck region comprises a third of the points closer to its geometrical center, a central region with the second third of points, and a dome region with the reminder (Fig. 3b). 


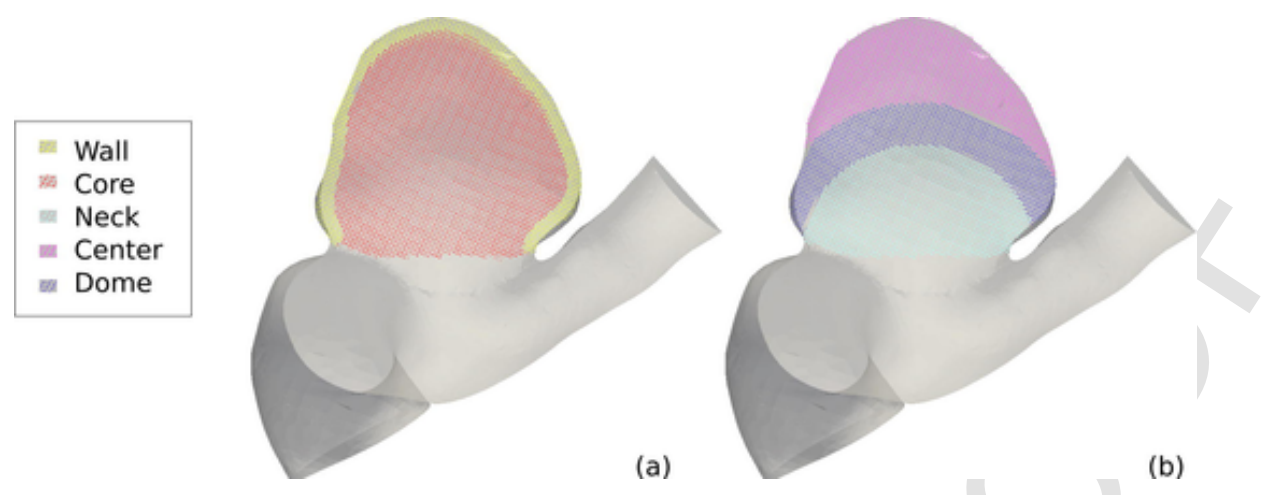

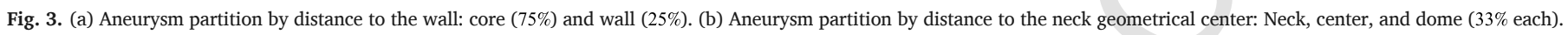

\subsection{Time}

Reduction of computational cost is perhaps the main goal of PM for modelling FD, which is expected to reduce computational load while easing the mesh generation process. Therefore, to produce a fair comparison, each simulation was run on the same processor, an Intel(R) Xeon(R) E5-2650 v4 CPU on a single thread.

\subsection{Mesh generation}

Unstructured volumetric meshes were created with snappyHexMesh tool. An additional level of refinement, doubling the number of elements, was done for each patient to assess discretization size. All models were then simulated on the refined meshes. The probed velocities on each model between the coarser and the refined mesh were compared. A relative error for each probed position $\left(D_{p}\right)$ between refinements for each model was calculated as:

$D_{p}=100 \cdot \frac{\left|U_{r}-U_{c}\right|}{U_{r}}$

where $U_{r}$ is the velocity probed on the refined mesh and $U_{c}$ is the velocity probed for the coarse mesh. There was an average relative error between probes of $0.93 \%$ and a standard deviation of $0.77 \%$. The untreated case and PM models had $1.8 \times 10^{6}$ elements, while BCM meshes had $7.3 \times 10^{6}$ elements, in average. This difference is due to the need for a more refined mesh close to the wires. While approaching a FD wire, the refinement level is doubled every two cells, until cell size becomes at least $t / 4$.

\section{Results}

\subsection{Velocity}

For all probes positions, velocity was compared between every PM model and BCM. The normalised velocity distributions are shown in Fig. 4. The dashed line represents a perfect match between the each PM model (separated by colour) and BCM. Points below this line were underestimated, while points above the line correspond to overestimated velocities by the PM model. M1 and M2 show bigger velocities than BCM in most of the cases. Velocities differ between this two models in patient 12, and lower for M1 in patients 01 and 14. On the right side of the dashed line, are most of A velocity probes. This means the model predicts lower velocities than BCM. There are exceptions in patients 03 and 08 , were velocities are larger, and patient 12 were values are more spread. Closer to the dashed line are R1 and R2, with a better resemblance of BCM than the other PM models. These differences between any PM model $m$ and BCM are presented in Table 1 , and computed as

$$
D_{m}=\frac{K E_{B C M}-K E_{m}}{K E_{B C M}}
$$

where $K E_{m}$ is the kinetic energy for the model $m$. The table shows how much bigger (positive values) or smaller (negative values) than BCM is the kinetic energy for each model $m$.

To quantify the similarity between PM models and BCM, the non parametric Kolmogorov-Smirnov test was used. The velocity distribution of each patient with each PM model was compared to the velocity distribution of the same geometry using BCM. Because blood motion presents different behaviour in different regions of the aneurysm, the tests are run in blocks by region. Also, data is grouped by flow type, namely: inertia-driven and shear-driven. The resultant statistics are shown in Fig. 5, showing similar behaviour in all regions. The untreated case has the largest difference to BCM, with a median larger than 0.4 in all regions. This differences are followed by $\mathbf{A}$ with a median between 0.15 and 0.54 . M1 and M2 show better results than A, with the homogeneous version rendering the best results. R2 clearly outperforms other models when compared to BCM, with a maximum statistic of 0.17 at the neck for shear-driven flow and 0.11 in the center region for inertia-driven. All models show better agreement near the wall (mean 0.16 , median 0.08). For shear-driven flow, the worst approximation is near the neck (mean 0.3 , median 0.2 ), while for inertia-driven flow this occurs at the center of the aneurysm (mean 0.3 , median 0.28 ).

\subsection{Wall Shear Stress (WSS)}

In Table 2 relative error values of WSS vs BCM over the aneurysm's wall for each patient are reported for each PM model. M1 results are between $48.4 \%$ lower and $54.7 \%$ higher than BCM, while M2 are in an even larger range, between $-45.1 \%$ and $+90.7 \%$. R1 only showed two mean WSS predictions larger than BCM, for patients $02(8.5 \%)$ and $07(5.8 \%$ ), while the results for the rest of the geometries fall short up to $-62 \%$. The WSS results that best resembled BCM were from R2, reporting values between $-57 \%$ and $+12 \%$ higher. A resulted, in most cases, a lower mean WSS than BCM. Only for Patient 01, A's WSS mean is $3.7 \%$ above, while for the remainder of geometries the mean values were lower up to $-70 \%$.

WSS scalar values of three different aneurysms are shown in Fig. 6. Patient 02 was selected because all PM models showed good agreement with BCM Fig. 4. In this case, there are no visible differences of WSS in treatment predictions. Velocities plotted for patient 03 in Fig. 4 are largely scattered. In Fig. 6 this results in different WSS patterns and impeachment zone. Finally patient 11 has, for each PM model, different velocity magnitudes but with similar patterns. This is seen as straight lines in Fig. 4, but at different angles. In Fig. 6 this is observed as similar impeachment zone but with different magnitudes. 


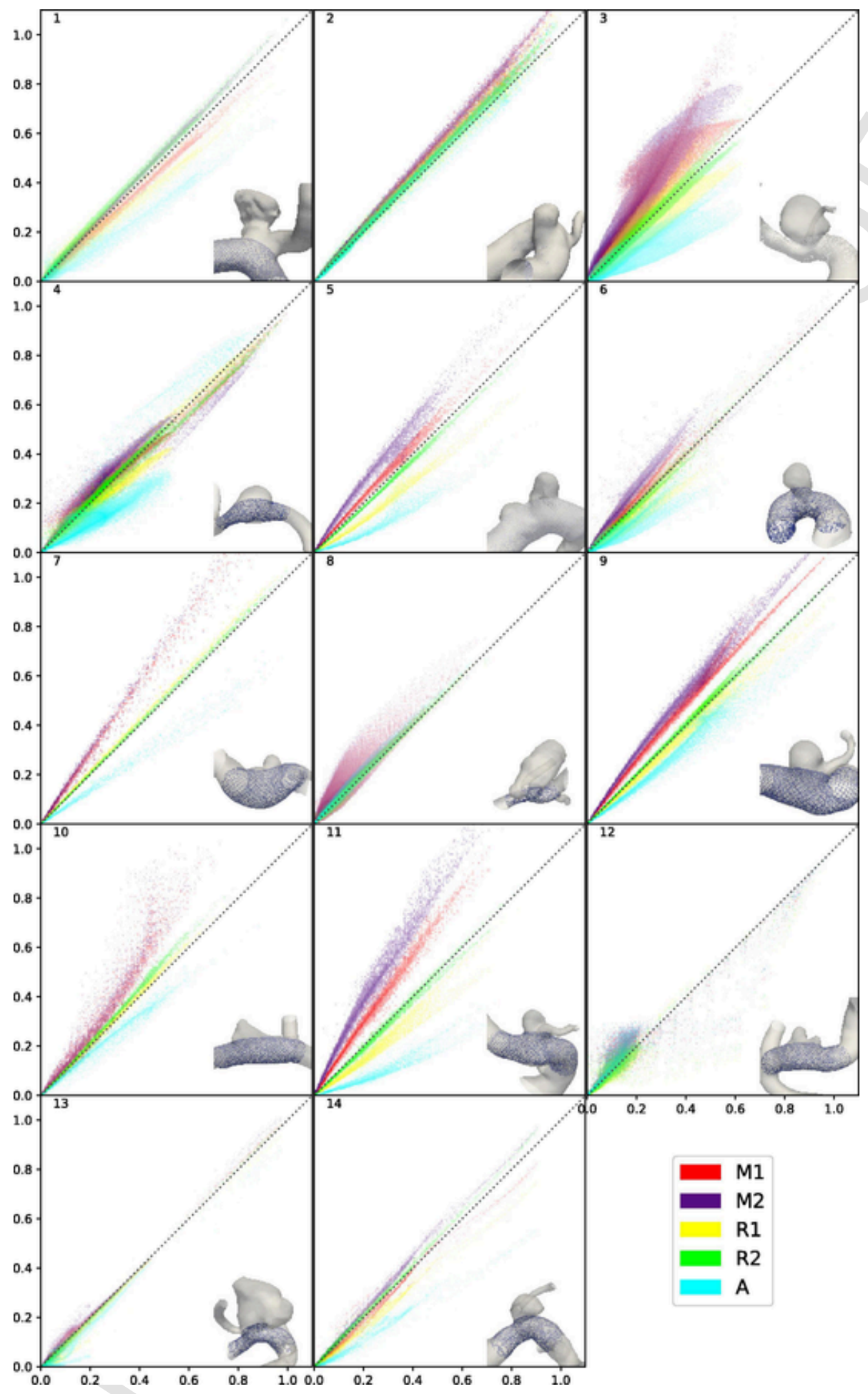

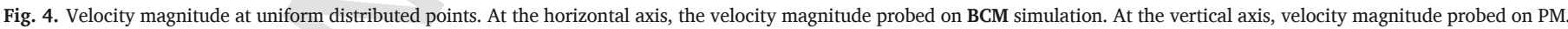
The dashed line represents a perfect match between the observed model and BCM.

\subsection{Computational time}

The time required to obtain a computational simulation with each model was measured. Fig. 7 shows time distributions disaggregated by processing step. In $\mathbf{B C M}$, meshing process is far more time consuming than the other steps. In A, porosity estimation step takes times between $110 \mathrm{~s}$ and $657 \mathrm{~s}$, with an outlier of $1686 \mathrm{~s}$, caused by a large neck aneurysm shown in Fig. 8a. The computation of PM model parameters for the rest of the models has negligible impact on overall time. Further, the simulation time difference between PM models is small, where simulations with larger intra-aneurysmal velocities take more time to converge.

Fig. 8b shows the time spend by each model versus the time of BCM, highlighting with straight lines the linear fit for each PM model. In general, PM models require less than a third of the time than BCM. 
Table 1

Kinematic energy variation between every model and BCM. The untreated case is also shown and compared to BCM, to highlight the change induced after treatment.

\begin{tabular}{|c|c|c|c|c|c|c|c|c|}
\hline Patient & Driver & $\mathrm{U}$ & $\mathrm{BCM}$ & M1 & M2 & $\mathrm{R} 1$ & $\mathrm{R} 2$ & A \\
\hline 01 & Inertia & $147.96 \%$ & $0.00 \%$ & $-10.38 \%$ & $12.99 \%$ & $-20.92 \%$ & $16.17 \%$ & $-49.97 \%$ \\
\hline 02 & Shear & $149.73 \%$ & $0.00 \%$ & $21.07 \%$ & $29.32 \%$ & $9.95 \%$ & $7.34 \%$ & $3.81 \%$ \\
\hline 03 & Inertia & $1073.26 \%$ & $0.00 \%$ & $117.84 \%$ & $163.47 \%$ & $-1.41 \%$ & $1.68 \%$ & $-65.75 \%$ \\
\hline 04 & Inertia & $242.05 \%$ & $0.00 \%$ & $3.21 \%$ & $17.84 \%$ & $-16.66 \%$ & $0.39 \%$ & $-36.54 \%$ \\
\hline 05 & Inertia & $906.65 \%$ & $0.00 \%$ & $20.73 \%$ & $72.53 \%$ & $-45.51 \%$ & $-11.89 \%$ & $-73.06 \%$ \\
\hline 06 & Shear & $303.85 \%$ & $0.00 \%$ & $11.33 \%$ & $54.03 \%$ & $-27.20 \%$ & $-8.73 \%$ & $-43.11 \%$ \\
\hline 07 & Shear & $654.66 \%$ & $0.00 \%$ & $100.56 \%$ & $114.53 \%$ & $15.92 \%$ & $9.13 \%$ & $-49.63 \%$ \\
\hline 08 & Shear & $624.77 \%$ & $0.00 \%$ & $55.43 \%$ & $64.58 \%$ & $2.21 \%$ & $4.38 \%$ & $10.93 \%$ \\
\hline 09 & Shear & $444.46 \%$ & $0.00 \%$ & $47.36 \%$ & $72.27 \%$ & $-12.37 \%$ & $2.00 \%$ & $-36.76 \%$ \\
\hline 10 & Shear & $1978.37 \%$ & $0.00 \%$ & $110.82 \%$ & $133.06 \%$ & $9.80 \%$ & $22.98 \%$ & $-30.95 \%$ \\
\hline 11 & Shear & $1983.06 \%$ & $0.00 \%$ & $108.74 \%$ & $218.30 \%$ & $-36.96 \%$ & $-3.47 \%$ & $-81.65 \%$ \\
\hline 12 & Shear & $131.09 \%$ & $0.00 \%$ & $-13.23 \%$ & $-7.59 \%$ & $-29.56 \%$ & $-26.01 \%$ & $-10.49 \%$ \\
\hline 13 & Shear & $92.48 \%$ & $0.00 \%$ & $6.10 \%$ & $20.08 \%$ & $-13.74 \%$ & $0.21 \%$ & $-29.33 \%$ \\
\hline 14 & Shear & $250.15 \%$ & $0.00 \%$ & $-9.55 \%$ & $26.85 \%$ & $-32.63 \%$ & $11.99 \%$ & $-64.77 \%$ \\
\hline MEAN & & $641.61 \%$ & $0.00 \%$ & $40.72 \%$ & $70.88 \%$ & $-14.22 \%$ & $1.87 \%$ & $39.80 \%$ \\
\hline
\end{tabular}

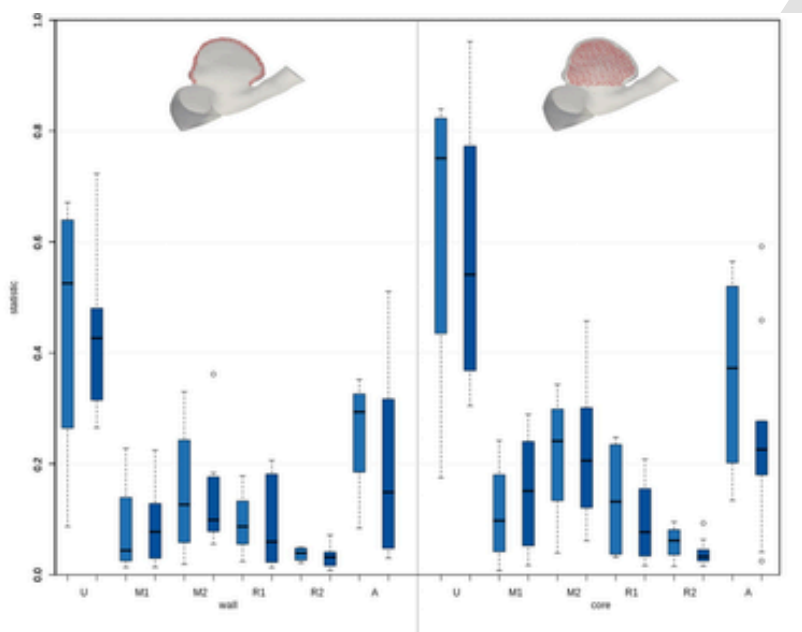

(a)

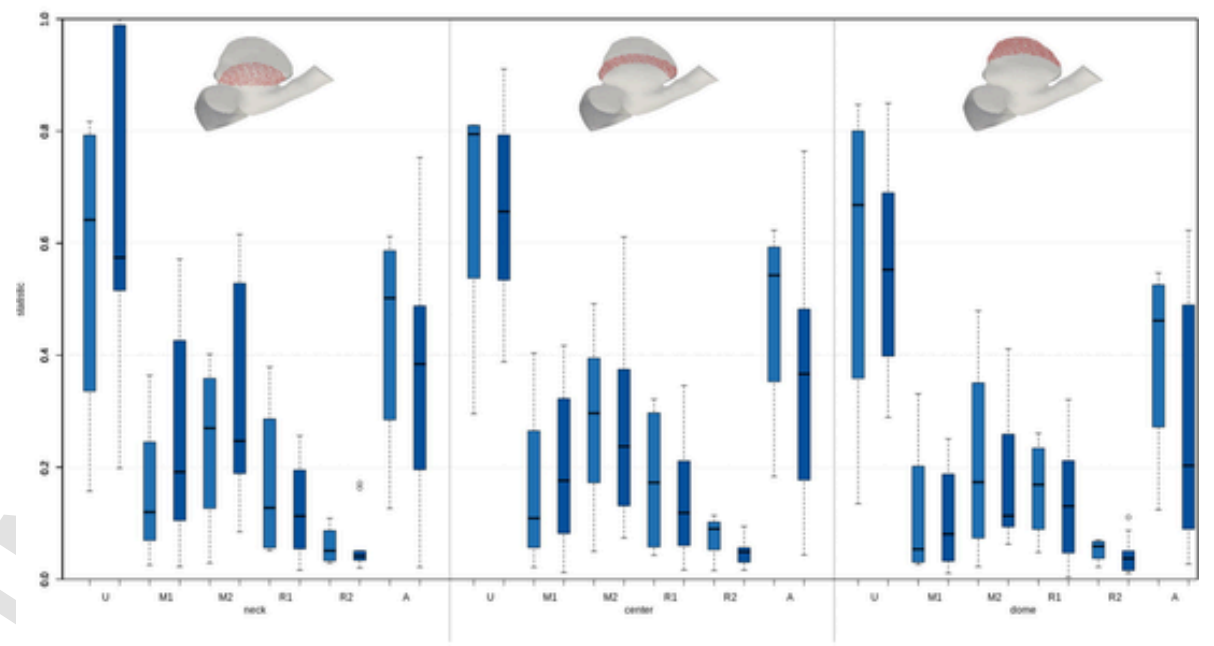

(c)
- Intertial driven

Shear driven

(b) 
Table 2

Relative error values of WSS vs BCM over aneurysm's wall on each patient.

Expand

\begin{tabular}{|c|c|c|c|c|c|c|c|c|}
\hline Patient & Driver & $\mathrm{U}$ & BCM & M1 & M2 & $\mathrm{R} 1$ & $\mathrm{R} 2$ & A \\
\hline 1 & Inertia & $75.35 \%$ & $0.00 \%$ & $-4.55 \%$ & $8.91 \%$ & $-11.19 \%$ & $11.85 \%$ & $-32.78 \%$ \\
\hline 2 & Shear & $119.09 \%$ & $0.00 \%$ & $16.70 \%$ & $22.96 \%$ & $8.57 \%$ & $5.75 \%$ & $3.73 \%$ \\
\hline 3 & Inertia & $376.89 \%$ & $0.00 \%$ & $54.69 \%$ & $75.55 \%$ & $-1.87 \%$ & $-0.25 \%$ & $-45.91 \%$ \\
\hline 4 & Inertia & $42.67 \%$ & $0.00 \%$ & $-40.75 \%$ & $-33.57 \%$ & $-51.99 \%$ & $-42.30 \%$ & $-67.25 \%$ \\
\hline 5 & Inertia & $340.41 \%$ & $0.00 \%$ & $12.31 \%$ & $40.44 \%$ & $-33.25 \%$ & $-9.91 \%$ & $-57.98 \%$ \\
\hline 6 & Shear & $27.84 \%$ & $0.00 \%$ & $-46.91 \%$ & $-33.00 \%$ & $-61.98 \%$ & $-53.83 \%$ & $-69.97 \%$ \\
\hline 7 & Shear & $203.65 \%$ & $0.00 \%$ & $40.50 \%$ & $45.68 \%$ & $5.86 \%$ & $2.95 \%$ & $-30.58 \%$ \\
\hline 8 & Shear & $157.20 \%$ & $0.00 \%$ & $-8.07 \%$ & $-5.33 \%$ & $-27.67 \%$ & $-27.42 \%$ & $-24.25 \%$ \\
\hline 9 & Shear & $245.88 \%$ & $0.00 \%$ & $30.02 \%$ & $45.66 \%$ & $-10.09 \%$ & $0.31 \%$ & $-28.82 \%$ \\
\hline 10 & Shear & $218.20 \%$ & $0.00 \%$ & $18.37 \%$ & $22.11 \%$ & $-5.69 \%$ & $-1.23 \%$ & $-23.04 \%$ \\
\hline 11 & Shear & $478.21 \%$ & $0.00 \%$ & $50.35 \%$ & $90.68 \%$ & $-24.23 \%$ & $-3.92 \%$ & $-61.28 \%$ \\
\hline 12 & Shear & $14.55 \%$ & $0.00 \%$ & $-48.40 \%$ & $-45.14 \%$ & $-60.18 \%$ & $-57.39 \%$ & $-47.06 \%$ \\
\hline 13 & Shear & $104.70 \%$ & $0.00 \%$ & $-3.41 \%$ & $16.44 \%$ & $-32.86 \%$ & $-12.30 \%$ & $-55.30 \%$ \\
\hline 14 & Shear & $164.32 \%$ & $0.00 \%$ & $-7.98 \%$ & $18.67 \%$ & $-23.28 \%$ & $12.08 \%$ & $-52.15 \%$ \\
\hline MEAN & & $183.50 \%$ & $0.00 \%$ & $4.49 \%$ & $19.29 \%$ & $-23.56 \%$ & $-12.54 \%$ & $-42.33 \%$ \\
\hline
\end{tabular}

A one-way ANOVA test between BCM and each model was done, resulting in $p<1 \times 10^{-9}$ for all models. As computational time increases in BCM, it also increases for PM models, but at a smaller rate, producing a speed-up increase of $11 \%$ between the least and the most time consuming patient. The relative difference between solution times increases for larger computational times. There is also a visible difference in computational time between $\mathbf{A}$ and the other PM models.

\section{Discussion}

Different modelling methodologies have been presented in the literature that are capable of representing FD simulations as a porous media with different levels of accuracy. Li, Yujie et al. analysed the sensitivity of PM coefficients and discovered that they greatly affect the aneurysmal flow patterns (Li et al., 2017). In this work, some of these models were used to simulate FD effect on hemodynamics in anatomically accurate vascular geometries. The results were then compared in terms of velocity, energy loss and WSS to assess numerical performance vs computational cost of each alternative. The performance was evaluated by comparing PM models to BCM. A method based on Kolmogorov-Smirnov was proposed and implemented to quantitatively asses the comparison.

The model proposed by Augsburger et al. (A) obtains the PM model coefficients using auxiliary simulations (Augsburger et al., 2011). The model consist on placing the portion of the device at the aneurysm neck into an ideal channel and performing simulations for tangential and orthogonal flow. Pressure drop is assessed for various $(n=6)$ velocities and fitted with a quadratic. Because of its experimental nature, the model can be applied to any kind of FD. On the other hand, a manual selection and cropping of the device portion at the aneurysms neck is required, involving additional time and manual processing. Also, the extra meshing and simulation steps on ideal channels take a large amount of computational effort and manual processing time (Mean time: 394s). In 12 out of 14 cases, velocities measured inside the aneurysm sac obtained from A model simulations were lower than BCM, as seen in Fig. 4. This ultimately leads to lower $K E$, and lower WSS when looking at the wall.

The model proposed by Morales et al. (M1) performs an analysis on the geometry of FD (according to its diameter and number of wires) to calculate average parameters to be used in the PM model coefficients (Morales and Bonnefous, 2014). In this model, the coefficients are calculated one time for each device size and diameter, independently of the patient anatomy. CFD results using this model result in higher ve- locities (40.7\% in average) inside the aneurysm sac than with BCM, confirming previous results (Dazeo et al., 2018a). This can be seen in Fig. 4, where the (red) point cloud corresponding to M1 remains over the bisector for most of the samples. This is due to the underestimation of the effect of the wire screen across the aneurysm neck on the flow, leading to a lesser pressure drop and loss of energy. An heterogeneous version (M2) of this model was implemented following the work of Yadollahi-Farsani et al. (2019). The porosity was calculated locally at each wire intersection from the angle between the wires and used to compute local values of $\mathbf{D}$ and $\mathbf{F}$. Those values were then linearly interpolated across the porous region of the simulation mesh. Because each deployment needs a specific porosity estimation, this alternative took $5 \%$ more time than the homogeneous version. However, results did not improve afterwards: K-S statistic increments in average $52 \%$ versus homogeneous model and velocities are in average $70.88 \%$ higher than BCM. Our interpretation of the results is that the main hypothesis of M1, were the model is adapted to complete FD wire screen, is overruled and locally neglected when moving to an heterogeneous setup. For instance, in the region of the aneurysm neck, the FD porosity $(\beta)$ is usually larger than the average. Because of this, local $d$ and $f$ coefficients are smaller and, consequently, the model as a has a lower resistance for M2 than for M1, leading to higher velocities inside the aneurysm sac. We observed that velocities in the M1 are higher than BCM. The reduction of $d$ and $f$ in M2 produces even higher velocities, leading to lower quality results. This observations are in line with the observations in previous work, were M1 under estimated PM resistance for higher mesh porosity (Dazeo et al., 2018a).

The model proposed by Raschi et al. (R1) uses the equations of hydraulic resistance for wire screens based on wire shapes, initially studied by Idelchick (Idelchik and Fried, 1986). This model obtains the parameters of an homogeneous porous region as a function of the geometrical variables describing the deployed braided mesh. R1, unlike M1, has custom porosity coefficients for each deployed device, which could be the cause for R1 better performance in comparison to M1: M1 K-S is in average 0.0228 units bigger than R1. This model has an average of $14.22 \%$ lower velocities than BCM, meaning that PM model coefficients and, ultimately, the wire screen effect on flow are overestimated. An heterogeneous variant (R2) was also implemented from this model. R2 K-S statistic has an average reduction of $27 \%$ compared to R1. Also, the velocities are in average only $1.87 \%$ higher than BCM. Contrary to M1, $\mathbf{R} 1$ attain lower velocities than $\mathbf{B C M}$, in general. Therefore, the same velocity increase caused by heterogeneity, makes 


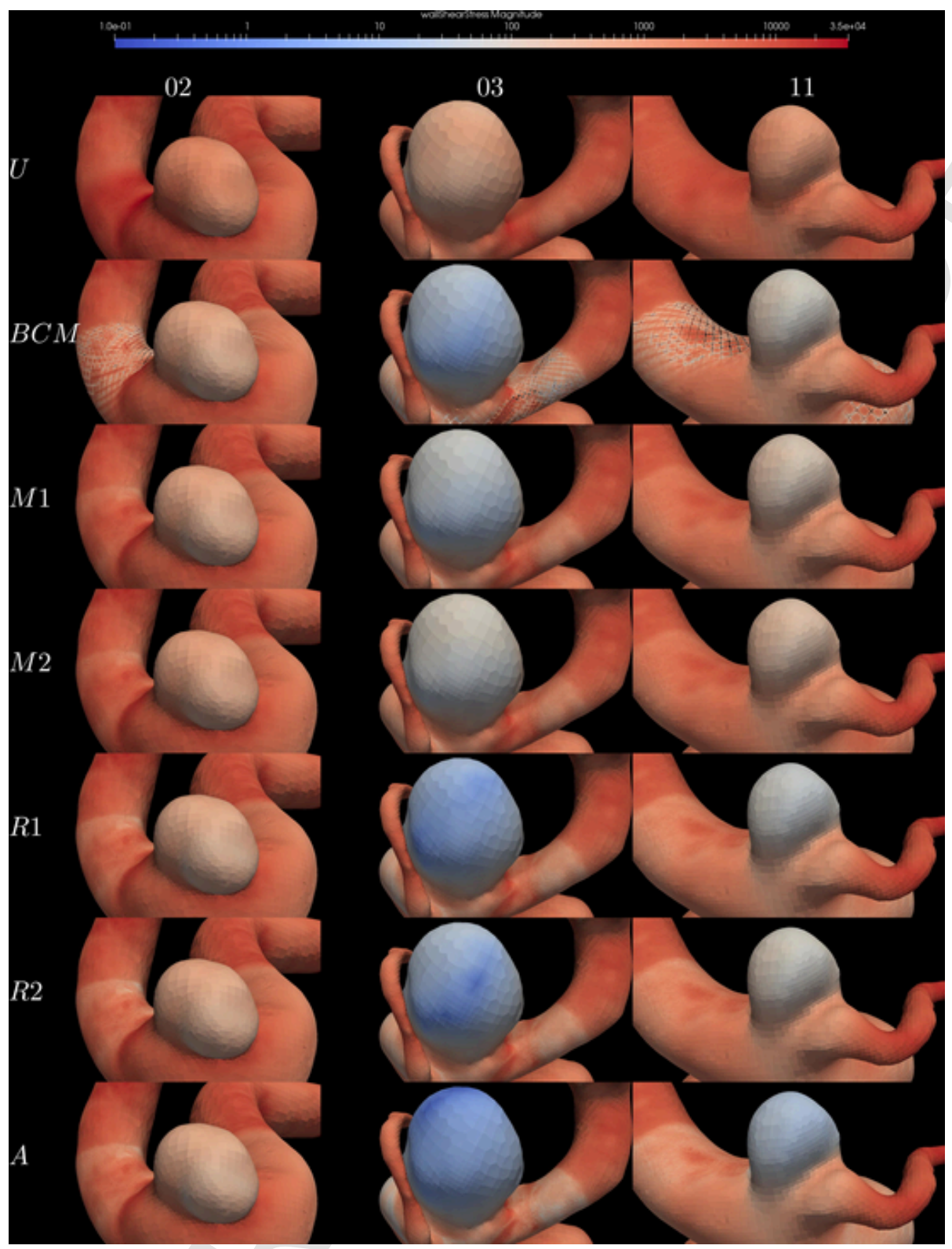

Fig. 6. Prediction of WSS magnitude for patients 02,03 and 11 .



Fig. 7. Porosity estimation, Meshing, Simulation and Total distribution times in seconds across patients of each model. Some models, as BCM and U, do not require porosity estimation. 


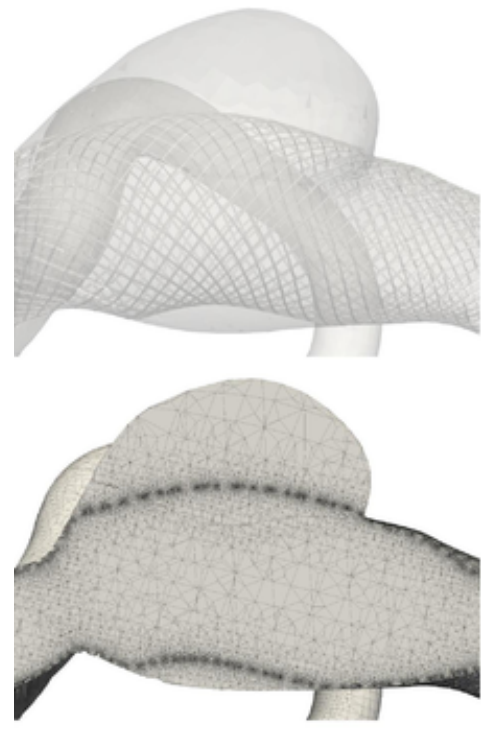

(a)

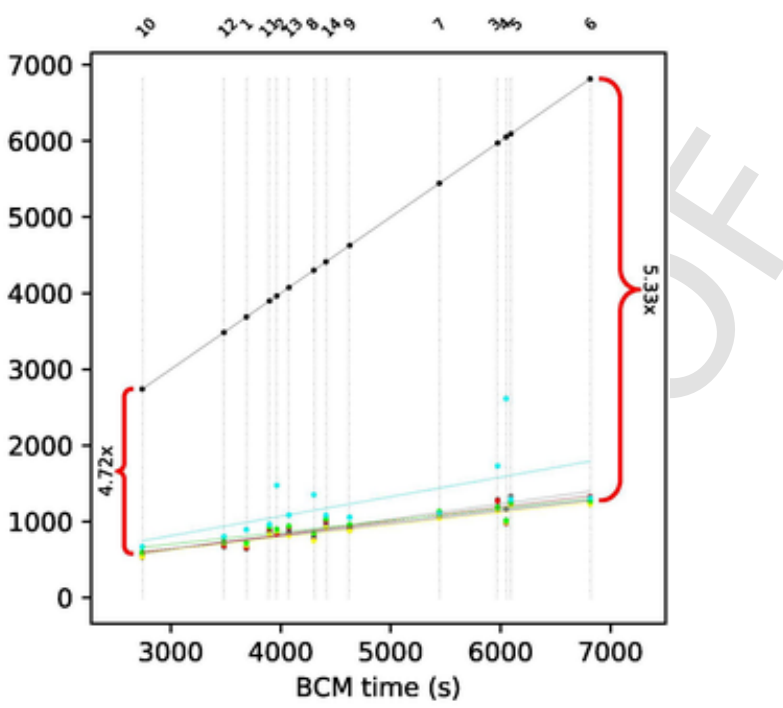

(b)

Fig. 8. (a) Aneurysm with a big neck. In A model, the neck size has a strong impact in PM model parameters. (b) Total time of each model divided by BCM time for each case.

R2 predictions closer to BCM than R1. Further, Idelchik's results considered in this study are aimed at flat rhombus shaped cells, as is the case for FDs, where each region has its own local geometric properties to be accounted for.

WSS is know to be implicated in the initiation, growth and rupture of aneurysms (Geers et al., 2017). Because the performance analysis was done by region, it is possible to assess how accurately each PM models performs in different regions within the aneurysm. This is of mayor importance in the computation of specific hemodynamic variables, such as WSS. PM models have good agreement with BCM near the wall region, were flow variables such as WSS and impeachment zone are of interest and typically calculated. Therefore, PM models are accurate and useful to approximately asses those variables. On the other hand, larger differences are observed near the neck, meaning that variables such as shear and vorticity near the neck will not be accurately assessed. This might be because of the "averaging" effect of PM models, which capture the global effect of the porous region of flow, at the expense of loosing local details. Still, while wall properties are commonly used in literature to study intra-aneurysmal flow after implantation of endovascular devices, the flow behaviour at the neck is not frequently observed in detail. We therefore consider that PM is a useful method for FD stent simulation when looking at specific regions. Still, care should be taken by the reader to understand the hypothesis and limitations of this method.

Finally, computational time was assessed to compare computational effort required by the different models. Computational time is drastically reduced when PM models are used. The average speedup using PM model ranged between 4.75x and 5.3x, being the speedup increased in cases with a larger baseline time (i.e., the longer takes to compute the BCM baseline, the larger the speedup will be). Also, there is a small difference in the total computation time between wire screen geometry based PM models (i.e., M1, M2, R1, R2). In average, the total times for each model ranged between $903 \mathrm{~s}(\mathbf{R 1})$ and $963 \mathrm{~s}(\mathbf{R 2})$. On the other hand, $\mathbf{A}$ has an overhead caused by coefficient estimation simulations, an average of $1244 \mathrm{~s}$ were porosity takes 394s. This model's auxiliary simulations are sensitive to aneurysm's neck size because the FD wire screen covering the aneurysm neck are used for coefficient estimation. Larger necks lead to larger wire screen portions, which have a larger and consequently more complex region where to generate a computa- tional mesh. This ultimately causes that PM model parameter computation to be longer.

In (Appanaboyina et al., 2009) authors analyse the effects of partial stent modelling. In their work, the portions that lay entirely on the vessel walls were removed. This model leads to an average reduction of $46.93 \%$ of mesh elements, with an average speedup of $2.22 x$, and an average difference in the variables of interest of $14.20 \%$. Differences between PM models, full and/or partial FD modelling could also be approached with this methodology in future studies.

Some limitations in this study must be remarked. First, the FD geometry used is a generic one, not representing any particular device available in the market. The results change when design variables are changed, yet they can be approached with the same methodology. Also, only a few PM models in literature were selected for comparison. Other PM models could be added by a pairwise comparison to BCM obtaining the K-S statistic. Finally, simulations are stationary, with constant inlet velocities. Geers et al. found than steady state simulations on laminar flow regimes provides similar hemodynamic information than flow rate wave-forms in comparison to the sensitivities given by patient specific uncertainties (Geers et al., 2014). However, pulsatile flow could entail inertia effects that might deserve a study on its own.

\section{Conclusions}

Using a PM strongly reduces mesh size and, therefore, computational loads. Augsburger et al. PM model has a time overhead mostly due to supplementary parameter estimation computation steps. Models based on geometrical properties can recreate specifics of FD hemodynamics more accurately with less computational effort and processing time. Spatially heterogeneous models, which can capture local variations of the mesh density and metal distribution, gave assorted results, depending on the model. R2 performance was closer to BCM than R1, but M2 had worst accomplishments than its heterogeneous counterpart M1. Finally, computational time is significantly reduced by all PM models.

\section{Declaration of Competing Interest}

The authors declare that they have no known competing financial interests or personal relationships that could have appeared to influence the work reported in this paper. 


\section{Acknowledgments}

This project was partly funded by PICT Start-up 2015-0006 and PICT 2016-0116 - FONCYT - ANPCYT of Argentina. N.D. is supported by CONICET PhD grant. The Titan Xp used for this research was donated by the NVIDIA Corporation. The financial support of these institutions is greatly appreciated.

\section{References}

Appanaboyina, S, Mut, F, Löhner, R, Putman, C, Cebral, J, 2009. Simulation of intracranial aneurysm stenting: techniques and challenges. Comput. Meth. Appl. Mech. Eng. 198, 3567-3582.

Augsburger, L, Reymond, P, Rufenacht, D, Stergiopulos, N, 2011. Intracranial stents being modeled as a porous medium: flow simulation in stented cerebral aneurysms. Ann. Biomed. Eng. 39, 850-863.

Castro, M, Putman, C, Sheridan, M, Cebral, J, 2009. Hemodynamic patterns of anterior communicating artery aneurysms: a possible association with rupture. Am. J. Neuroradiol. 30, 297-302.

Dazeo, N., Dottori, J., Boroni, G., Clausse, A., Larrabide, I., 2017. Flow diverter stents simulation with cfd: porous media modelling. inn: 12th International Symposium on Medical Information Processing and Analysis, International Society for Optics and Photonics. p. 101601F.

Dazeo, N, Dottori, J, Boroni, G, Larrabide, I, 2018. A comparative study of porous medium cfd models for flow diverter stents: advantages and shortcomings. Int. J. Numer. Meth. Biomed. Eng. 34, e3145.

Dazeo, N., Dottori, J., Boroni, G., Larrabide, I., 2018b. Heterogeneous porous media simulation. In: Mecánica Computacional. Argentine Association For Computational Mechanics. pp. 1173-1181.

Geers, A, Larrabide, I, Morales, H, Frangi, A F, 2014. Approximating hemodynamics of cerebral aneurysms with steady flow simulations. J. Biomech. 47, 178-185.

Geers, A, Morales, H, Larrabide, I, Butakoff, C, Bijlenga, P, Frangi, A, 2017. Wall shear stress at the initiation site of cerebral aneurysms. Biomech. Model. Mechanobiol. 16, 97-115.

Idelchik, I E, Fried, E, 1986. Handbook of Hydraulic Resistance. Hemisphere Publishing, New York, NY.

Jasak, H, Jemcov, A, Tukovic, Z, et al., 2007. Openfoam: A c ++ library for complex physics simulations. In: International Workshop on Coupled Methods in Numerical Dynamics. IUC Dubrovnik, Croatia, pp. 1-20.

Larrabide, I, Kim, M, Augsburger, L, Villa-Uriol, M C, Rüfenacht, D, Frangi, A F, 2012. Fast virtual deployment of self-expandable stents: method and in vitro evaluation for intracranial aneurysmal stenting. Med. Image Anal. 16, 721-730.

Larrabide, I, Villa-Uriol, M C, Cárdenes, R, Barbarito, V, Carotenuto, L, Geers, A J, Morales, H G, Pozo, J M, Mazzeo, M D, Bogunović, H, et al., 2012. Angiolab-a software tool for morphological analysis and endovascular treatment planning of intracranial aneurysms. Comput. Meth. Programs Biomed. 108, 806-819.

Larrabide, I, Aguilar, M, Morales, H, Geers, A, Kulcsár, Z, Rüfenacht, D, Frangi, A, 2013 Intra-aneurysmal pressure and flow changes induced by flow diverters: relation to aneurysm size and shape. Am. J. Neuroradiol. 34, 816-822.

Li, Y., Zhang, M., Verrelli, D.I., Yang, W., Chong, W., Ohta, M., Qian, Y., 2017. Sensitivity study on modelling a flow-diverting stent as a porous medium using computational fluid dynamics. In: 2017 39th Annual International Conference of the IEEE Engineering in Medicine and Biology Society (EMBC). IEEE. pp. 3389-3392.

Li, S, Chopard, B, Latt, J, 2019. Continuum model for flow diverting stents in 3d patient-specific simulation of intracranial aneurysms. J. Comput. Sci. 38, 101045.

Morales, H.G., Bonnefous, O., 2014. Modeling hemodynamics after flow diverter with a porous medium. In: 2014 IEEE 11th International Symposium on Biomedical Imaging (ISBI). IEEE. pp. 1324-1327.

Morales, H G, Larrabide, I, Geers, A J, Aguilar, M L, Frangi, A F, 2013. Newtonian and non-newtonian blood flow in coiled cerebral aneurysms. J. Biomech. 46, 2158-2164.

Moyano, R K, Fernandez, H, Macho, J M, Blasco, J, San Roman, L, Narata, A P, Larrabide, I, 2017. A robustness test of the braided device foreshortening algorithm. In: 13th International Conference on Medical Information Processing and Analysis. International Society for Optics and Photonics, p. 105721H.

Narata, A P, Blasco, J, Roman, L S, Macho, J M, Fernandez, H, Moyano, R K, Winzenrieth, R, Larrabide, I, 2018. Early results in flow diverter sizing by computational simulation: quantification of size change and simulation error assessment. Oper. Neurosurg. 15, 557-566.

Raschi, M, Mut, F, Löhner, R, Cebral, J, 2014. Strategy for modeling flow diverters in cerebral aneurysms as a porous medium. Int. J. Numer. Meth. Biomed. Eng. 30, 909-925.

Reneman, R S, Arts, T, Hoeks, A P, 2006. Wall shear stress-an important determinant of endothelial cell function and structure-in the arterial system in vivo. J. Vasc. Res. 43, 251-269.

Yadollahi-Farsani, H, Scougal, E, Herrmann, M, Wei, W, Frakes, D, Chong, B, 2019. Numerical study of hemodynamics in brain aneurysms treated with flow diverter stents using porous medium theory. Comput. Meth. Biomech. Biomed. Eng. 1-11. 